\title{
The Apache Point Observatory Galactic Evolution Experiment (APOGEE)
}

\author{
Ricardo P. Schiavon ${ }^{1}$ \& Steven R. Majewski ${ }^{2}$ \\ ${ }^{1}$ Gemini Observatory, 670 N. A’Ohoku Place, Hilo, HI 96720, USA \\ email: rschiavon@gemini.edu \\ ${ }^{2}$ University of Virginia, P.O. Box 400325, Charlottesville, VA 22904-4325, USA \\ email: srm4n@virginia.edu
}

\begin{abstract}
The Apache Point Observatory Galactic Evolution Experiment (APOGEE) is a large scale, high-resolution, near-infrared spectroscopic survey of Milky Way stellar populations and one of the four experiments in the Sloan Digital Sky Survey III (SDSS-III). APOGEE will be based on a new multi-fiber cryogenic spectrograph, currently under construction, expected to begin survey observations on the $2.5 \mathrm{~m}$ Sloan telescope in the Spring of 2011. APOGEE will measure high-precision radial velocities and elemental abundances for $\sim 15$ elements for $\sim 10^{5}$ stars, and is expected to shed new light on the processes that led to the formation of the Galaxy.
\end{abstract}

Keywords. Stars: abundances, stars: kinematics, Galaxy: abundances, Galaxy: bulge, Galaxy: disk, Galaxy: kinematics and dynamics, Galaxy: evolution

\section{The Survey}

APOGEE aims to constrain models for the formation of the Galaxy by measuring accurate elemental abundances and radial velocities for a very large sample of Galactic stars. To achieve this goal, APOGEE will use a significant fraction of the SDSS-III bright time during a 3-year period to collect high-resolution $(R \sim 30,000)$, high $\mathrm{S} / \mathrm{N}$ ( $\gtrsim 100 /$ pixel) $H$-band spectra for $\sim 10^{5}$ stars across all components of the Galaxy, including the bulge, halo, and both thin and thick disks. Taking advantage of low extinction in the NIR $\left(A_{H} / A_{V}=0.16\right)$, APOGEE will be the first survey to pierce through dust obscuration and provide a vast, uniform database of chemical abundances and radial velocities for stars across all Galactic components. The survey will be conducted with a dedicated, 300-fiber, cryogenic spectrograph operating contiguously from $1.51 \mu \mathrm{m}$ to $1.68 \mu \mathrm{m}$ and coupled to the Sloan 2.5-m telescope at APO. APOGEE will rely on the Two Micron All-Sky Survey (2MASS) to select targets down to a typical flux limit of $H$ $<12-14$, and therefore, unlike most spectroscopic surveys, it does not require an extensive and onerous photometric survey phase. For more details, see Allende Prieto et al. (2008).

\section{The Data}

With its high resolution and $S / N$, APOGEE will determine for its vast sample of stars both precision radial velocities (with accuracy better than $0.5 \mathrm{~km} \mathrm{~s}^{-1}$ ) and accurate ( 0.1 dex) abundance measurements spanning numerous chemical species, including $\mathrm{C}$, $\mathrm{N}, \mathrm{O}$ and $\mathrm{Fe}$, most of the $\alpha$-elements, odd-Z elements, and iron-peak elements. APOGEE's massive database will provide a standard against which chemodynamical models for the formation of the Galaxy can be tested. Moreover, the huge number of homogeneous and 

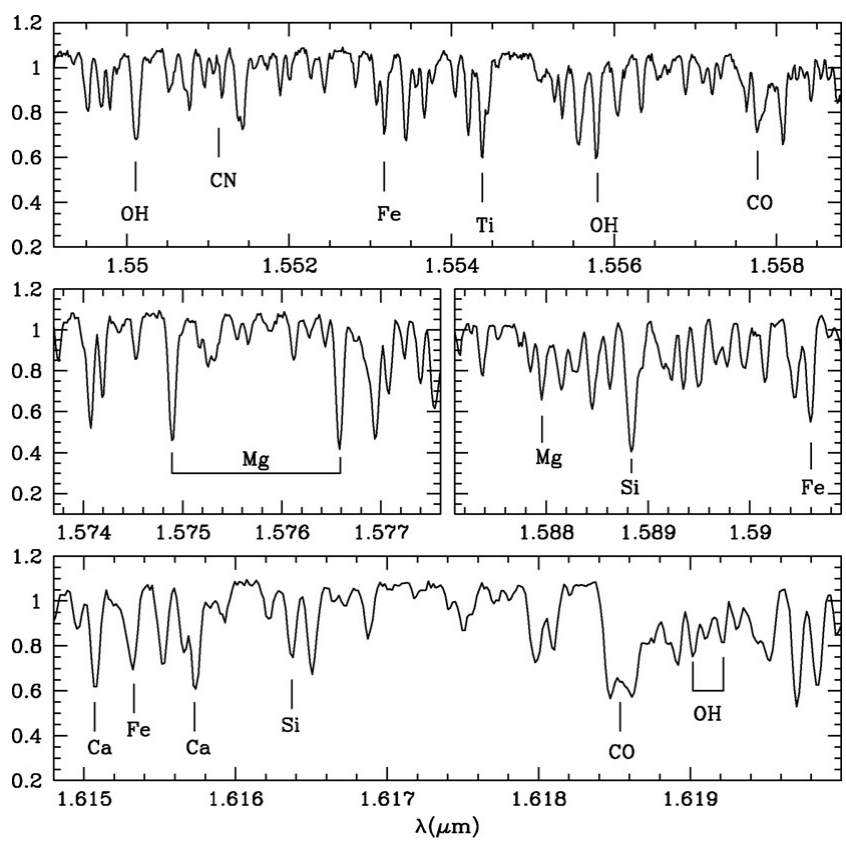

Figure 1. Simulated APOGEE spectra at $R=30,000$ and $S / N=100 /$ pixel, in selected spectral regions. Indicated are positions of a few of the absorption lines that will be used for measuring some of APOGEE key elemental abundances, such as Fe, C, N, O, Ca, Si, Mg, and Ti.

consistently-calibrated $H$-band spectra, stellar parameters, and elemental abundances will constitute a vast resource for future stellar population synthesis studies.

\section{Science Goals}

Among the main scientific objectives of APOGEE are: (1) to measure in an unbiased, uniform manner and with statistically large samples of stars, the metallicity and abundance pattern distributions for stars in all components of the Galaxy; (2) to constrain the processes of star formation, feedback, chemical mixing and chemical evolution in the Milky Way with sensitivity to numerous nucleosynthetic pathways; (3) to survey the dynamics of the Galaxy, particularly in the bulge and disk, where constraints on the nature and influence of the Galactic bar and spiral arms will be defined; (4) to look for chemical and dynamical evidence for Galactic substructure from accretion, thought to be an important contributor to Galactic stellar populations, in the context of hierarchical formation models, and (5) to identify low-latitude halo sub-structure, such as the Monoceros stream, and study the kinematics and chemical composition of known tidal streams; (6) to conduct a legacy survey of Galactic open clusters, which will constrain the history of star formation and chemical enrichment of the Galactic disk.

We thank APOGEE team members C. Allende Prieto, D. Bizyaev, P. Frinchaboy, L. Girardi, F. Hearty, J. Holtzman, J. Johnson, M. Shetrone, M. Skrutskie, and J. Wilson for their inestimable contribution.

\section{Reference}

Allende Prieto, C. et al. 2008, AN, 329, 1018. 